conducting in San Francisco, directly evaluates the frequency of occult HIV infection among blood donations found to be negative on all required tests. To facilitate the evaluation of a large number of donors by culture for HIV and the polymerase chain reaction, we combined the lymphocytes separated from each donation into pools of 50. As of May 1, 1989, the culture analysis was complete for 913 pools containing cells from 45,650 antibody-negative donations. The results were presented at the 1989 international conference on the acquired immunodeficiency syndrome (AIDS) in Montreal. ${ }^{5} \mathrm{~A}$ single pool was repeatedly positive for HIV on culture, and this result was confirmed by the polymerase chain reaction. ${ }^{6}$ After adjusting for the sensitivity of our detection systems by means of a group-testing estimate, ${ }^{6}$ we calculated a 1 -in- 42,400 point estimate of the frequency at which an HIV-infected donor is missed by current screening measures (upper 95 percent confidence bound, 1 in $8800)$.

These preliminary results ${ }^{4,5}$ provide direct data to validate the risk estimates based on statistical models. The data in Table 1 collectively put in perspective the risk of HIV transmission after transfusion of fully screened blood. Although certainly not zero, the level of risk is moving progressively closer to the desired goal - the safest blood supply that science and technology can realistically provide.

Michael Busch, M.D., Ph.D. Bernard Eble, Ph.D. David Heilbron, Ph.D. Girish Vyas, Ph.D.

San Francisco, CA 94143

Irwin Memorial Blood Center

1. Ward JW, Holmberg SD, Allen JR, et al. Transmission of human immunodeficiency virus (HIV) by blood transfusions screened as negative for HIV antibody. N Engl J Med 1988; 318:473-8.

2. Kleinman $\mathbf{S}$, Secord K. Risk of human immunodeficiency virus (HIV) transmission by anti-HIV negative blood: estimates using the lookback methodology. Transfusion 1988; 28:499-501.

3. Imagawa DT, Lee MH, Wolinsky SM, et al. Human immunodeficiency virus type 1 infection in homosexual men who remain seronegative for prolonged periods. N Engl J Med 1989; 320:1458-62.

4. Cohen ND, Muñoz A, Reitz BA, et al. Transmission of retrovirus by transfusion of screened blood in patients undergoing cardiac surgery. N Engl J Med 1989; 320:1172-6.

5. Busch MP, Eble B, Ulrich $P$, et al. Prospective evaluation of contemporary screened blood donors for occult HIV infection. In: Abstracts of the Fifth International Conference on AIDS, Montreal, June 4-9, 1989. Montreal: International Development Research Center, 1989:46.

6. Sobel M, Elashoff RM. Group testing wth a new goal, estimation. Biometrika $1975 ; 62: 181-93$.

The above letters were referred to the authors of the article in question, who offer the following reply:

To the Editor: Dr. Mintz is correct in stating that our study does not include data from certain major metropolitan areas with populations having a high prevalence of HIV-1. The American Red Cross collects blood throughout the United States in an overall area containing 50 percent of the nation's population. The 51 American Red Cross regions included in our study contained populations with high, medium, and low prevalences of HIV-1. On the high end are Atlanta, Los Angeles, and Miami, although none of these have a rate as high as those reported for New York and San Francisco. However, the rates of AIDS reported by the Centers for Disease Control* $^{*}$ do not necessarily correlate with the rates of HIV-1 infection in the blood supply, particularly in large metropolitan areas. In addition to the efficacy of donor recruitment and screening methods, areas such as New York obtain a large portion of their blood supply from areas with lower prevalence, such as suburban communities. Nonetheless, the point inferred by Dr. Mintz - that the risk of receiving HIV-1 from locally collected blood is greater in highprevalence than in low-prevalence areas - appears valid.

Our model implies that the risk of infection increases with the

*Centers for Disease Control. HIV/AIDS surveillance report. December 1989:6-7. incidence of HIV-1 infection and that the incidence rate itself is a function of seroprevalence. Differences between our estimates of infection risk and those cited by Dr. Busch and his colleagues were discussed in our article, with the exception of the new, culturebased data. With regard to the latter results, it should be stressed that estimates based on a single positive finding inevitably have a very wide confidence interval. Given that the risk estimate presented in our article represents an average for a large number of blood centers throughout the United States, and that we would expect to find local variations in HIV-1 prevalence, we do not see any inconsistency between the various estimates. Indeed, it is encouraging to find that the estimates produced by our window-period model are reasonably consistent with estimates obtained by other approaches. Measures to reduce the frequency of transfusion-transmitted HIV infection clearly continue to be effective even in areas with a high prevalence of HIV infection, such as San Francisco.

Paul D. Cumming, Ph.D.

Vienna, VA 22182 Talisman Ltd./Physician International

Buffalo, NY 14260

Edward L. Wallace, Ph.D State University of New York

New York, NY 10022

Julian B. SchorR, M.D. Immuno-U.S

Roger Y. Dodd, Ph.D

Washington, DC 20006 American Red Cross

\section{MATCHING FOR AGE IN RENAL TRANSPLANTATION}

To the Editor: Transplantation is increasingly available for elderly patients with kidney failure. ${ }^{1}$ Likewise, there is a trend toward increasing use of organs taken from older donors (i.e., over 50 years of age). ${ }^{2}$ This is occurring despite evidence of relatively poor results with respect to graft survival with older donors as compared with younger donors, even with good HLA matching. One means of exploiting this important source of organs more efficiently might be to take more account of the ages of donor and recipient relative to each other. Surprisingly, there is no evidence in the literature of any attempt to document the possible benefits of such matching for age or to recommend optimal donor-recipient age relationships.

To examine this question, we considered graft survival in 274 recipients of first transplants from cadavers between 1983 and 1987 at two centers in the United Kingdom: Leicester and Newcastle. All patients received cyclosporine for immunosuppression, and the overall one-year rate of graft survival was 85 percent, with no significant difference between centers. The age of the recipient did not affect the results, but there was some evidence of an effect of the donor's age, with a one-year graft survival of 78 percent in 57 cases involving donors over 50, as compared with 87 percent in the 217 cases involving younger donors, although the difference was not statistically significant $(P \approx 0.1)$. Taken together, however, the difference in age between the donor and the recipient had a strong influence on graft survival $(\mathrm{P}<0.01)$, as shown in Table 1 .

This effect was present without respect to the donor's age and also when nonimmunologic failures or death with a functioning graft were included as failures. The inference is that improved results can be obtained with older donors by transplanting their kidneys to recipients of at least equal age, rather than using the organs in younger recipients.

How does the effect of age considerations compare in importance

Table 1. Graft Survival One Year after Kidney Transplantation, According to the Difference in Age between the Donor and the Recipient.

\begin{tabular}{lccc}
\hline \hline & $\begin{array}{c}\text { DONOR }>5 \text { YR } \\
\text { Younger THAN } \\
\text { RECIPIENT }\end{array}$ & $\begin{array}{c}\text { DONOR WITHIN 5 YR } \\
\text { OF RECIPIENT }\end{array}$ & $\begin{array}{c}\text { DONOR >5 YR } \\
\text { OLDER THAN } \\
\text { RECIPIENT }\end{array}$ \\
No. of patients & 149 & 60 & 65 \\
Graft survival (\%) & 91 & 80 & 75 \\
\hline
\end{tabular}


with antigen matching? Our evidence is that the effect is at least comparable in magnitude with HLA-DR matching. Only two risk factors were declared significant $(\mathrm{P}<0.05)$ in a multifactor analysis of our results: the age difference described above and the number of DR-antigen mismatches. One-year graft survival was 91 percent in 95 patients with no DR mismatches, 83 percent in 29 patients with one mismatch, and 78 percent in 50 patients with two mismatches, giving a slightly narrower range of best to worst (13 percent) than the 16 percent range for the effect of age spread. In addition, a poor DR match (one or more mismatches) was associated with good oneyear graft survival if the effect of age was favorable, whereas if the age effect was unfavorable (donor greater than five years older than recipient), then HLA-DR matching did not improve the results.

A possible explanation for this age-matching effect may be the combined effect of an age-related decline in the immune response of the recipient and a similar age-related decline in function of the donor's renal tissue. In persons of both sexes over 30 years of age, the glomerular filtration rate declines linearly with time at a rate of $13 \mathrm{ml}$ per minute per decade. ${ }^{3}$ The limited functional reserve of kidneys from older donors would be further exhausted by insults that could include rejection episodes and cyclosporine nephrotoxicity. Because immunologic response decreases with advancing age, ${ }^{4}$ the allograft response of an older recipient to an organ from a donor of comparable age may be expected to be less than that of a younger recipient.

Age matching could perhaps improve the results of transplantation, particularly when kidneys from older donors are used.

\section{P.K. Donnelly}

R. Henderson University of Leicester School of Medicine

\section{Leicester LE5 4PW, England}

transplantation in older Howard RJ, Pfaff WW, Salomon D, et al.
patients. Transplant Proc 1989; 21:2020-1.

2. Foster MC, Wenham PW, Rowe PA, et al. Use of older patients as cadaveric kidney donors. Br J Surg 1988; 75:767-9.

3. Wesson LG Jr. Physiology of the human kidney. New York: Grune \& Stratton, 1969:96-108.

4. Makinodan T, Perkins EH, Chen MG. Immunological activity of the aged. Adv Geronotol Res 1971; 3:171-98.

To the Editor: We have analyzed data from the Eurotransplant data base in a way similar to that proposed by Donnelly and Henderson. Our data comprised information on 6397 patients receiving their first kidney transplants from unrelated nonliving donors from January 1984 through December 1987 and followed up through October 1988. We, too, found a significant effect $\langle P=0.0001$ by the log-rank test) of age difference, as shown in Table 1 (in which the deaths of patients with functioning grafts are included as graft failures).

The difference in graft survival increased with time and was statistically significant over the 4.5 -year period analyzed. We have also produced a prognostic index that takes other relevant factors into account and calculates an overall risk score on the basis of the contribution of each variable in a multifactorial Cox regression analysis. The age difference remained independently significant within this model $(P=0.01)$. Furthermore, we observed a strong

Table 1. Graft Survival after Kidney Transplantation over Time, According to the Difference in Age between the Donor and the Recipient.

\begin{tabular}{lccc}
\hline \hline ChARACTERISTIC & $\begin{array}{c}\text { DONOR }>5 \text { YR } \\
\text { YouNGER THAN } \\
\text { RECIPIENT }\end{array}$ & $\begin{array}{c}\text { DONOR WITHIN 5 YR } \\
\text { OF RECIPIENT }\end{array}$ & $\begin{array}{c}\text { DONOR }>5 \text { YR } \\
\text { OLDER THAN } \\
\text { RECIPIENT }\end{array}$ \\
$\begin{array}{l}\text { No. of patients } \\
\text { Graft survival (\%) }\end{array}$ & 3732 & 1301 & 1364 \\
1 year & 85.8 & 84.4 & \\
2 years & 81.5 & 79.4 & 82.1 \\
3 years & 76.7 & 75.6 & 69.3 \\
\hline
\end{tabular}

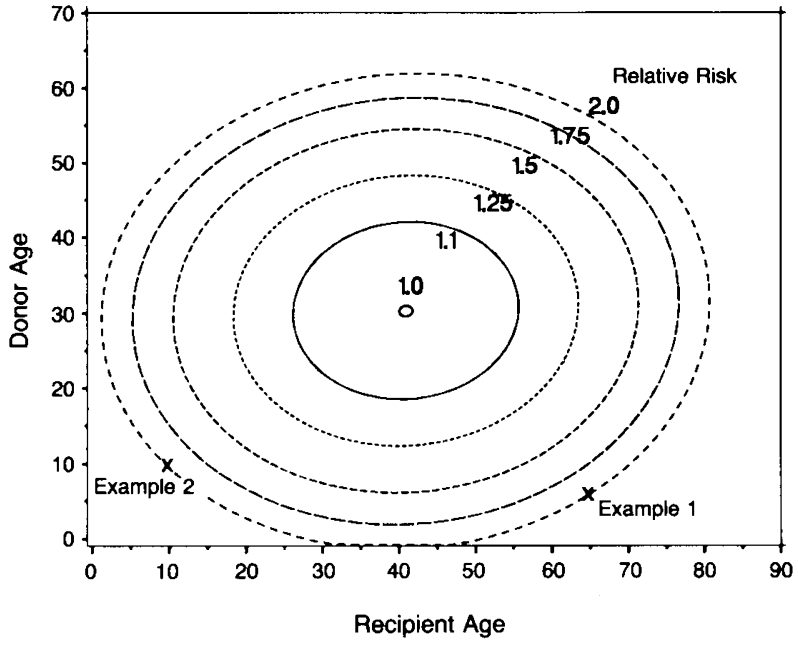

Figure 1. Contour Plots of the Relative Risk of Graft Failure, According to the Ages of Donor and Recipient.

and independent effect of HLA-DR matching on survival to one year after transplantation, which was present in all combinations of age difference between donors and recipients, although it had a greater effect when the donor was younger than the recipient $(P=0.0001)$ or within five years of the recipient's age $(P=0.007)$ than when the donor was more than five years older $(\mathrm{P}=0.05)$.

In previous work with the age groups of donors and recipients as separate variables, we had identified the age groups that were at particularly high risk - donors under 5 or over 55 years of age and recipients ranging in age from 0 to 15 years. We were interested in comparing the relative weights of analyzing age by the two approaches and concluded, using a Cox-model analysis, that the separate-age-group method was more predictive than the age-difference method. To indicate a possible reason, we developed Figure 1, which shows the contribution to the relative risk of graft failure of discrete donor and recipient ages, based on a model in which the other prognostic factors are allowed for sex of the donor and recipient, HLA match, percent highest panel-reactive antibody, cold ischemic period, recipient's blood group, whether the recipient was diabetic, and transplant center. The risk is lowest for donors about 30 and recipients about 40 years of age. The further one moves from this combination, the higher the relative risk of graft failure. Thus, a kidney from a donor 5 years old that is transplanted to a recipient 65 years old has a very poor prognosis (Example 1, Fig. 1). This is not explained by the age-difference method, which would have predicted a good prognosis, since the donor is more than 5 years younger than the recipient. As a second example, a kidney from a 10-year-old donor transplanted to a 10-year-old recipient also has a poor prognosis, even though they are matched for age (Example 2, Fig. 1). Such observations have been reported previously by Groenewoud et al. ${ }^{1}$ and Ploeg et al. ${ }^{2}$

We found the results of Donnelly and Henderson very interesting, and they stimulated us to look at the donor-recipient age difference in more detail. We believe that similar age groups may have a good prognosis but would advise against a general application of the rule that "young donors to old recipients have the best prognosis."

J. Thorogood, M.Sc. G.G. Persijn, M.D., Ph.D.

F.A. Zantvoort, M.D.

2300 RC Leiden, the Netherlands Eurotransplant Foundation

2312 AV Leiden, the Netherlands

J.C. van Houwel.ingen, Ph.D. Leiden University

2300 RC Leiden, the Netherlands

J.J. van Rood, M.D., Ph.D. Leiden University Hospital 